\title{
Assessment of the performance of High Luminosity LHC operational scenarios: integrated luminosity and effective pile-up density
}

\begin{tabular}{|r|l|}
\hline Journal: & Canadian Journal of Physics \\
\hline Manuscript ID & cjp-2018-0291.R1 \\
\hline Manuscript Type: & Article \\
\hline Author: & 18 -Jul-2018 \\
\hline Complete List of Authors: & $\begin{array}{l}\text { Medina Medrano, Luis; Universidad de Guanajuato, División de } \\
\text { Ciencias e Ingenierías; European Organization for Nuclear } \\
\text { Research } \\
\text { Tomas, R.; European Organization for Nuclear Research } \\
\text { Arduini, Gianluigi; European Organization for Nuclear Research } \\
\text { Napsuciale Mendívil, Mauro; Universidad de Guanajuato, } \\
\text { División de Ciencias e Ingenierías }\end{array}$ \\
\hline Keyword: & $\begin{array}{l}\text { Effective pile-up density, Luminosity, Integrated luminosity, HL- } \\
\text { LHC, High Luminosity LHC }\end{array}$ \\
\hline $\begin{array}{r}\text { Is the invited manuscript } \\
\text { for consideration in a } \\
\text { Special Issue? : }\end{array}$ & Not applicable (regular submission) \\
\hline & \multicolumn{2}{|l}{} \\
\hline
\end{tabular}




\title{
Assessment of the performance of High Luminosity LHC operational scenarios: integrated luminosity and effective pile-up density
}

\author{
L. Medina,${ }^{1,2, *}$ R. Tomás, ${ }^{2}$ G. Arduini, ${ }^{2}$ and M. Napsuciale ${ }^{1}$ \\ ${ }^{1}$ División de Ciencias e Ingenierías, Universidad de Guanajuato, \\ Loma del Bosque 103, C.P. 37150, León, Mexico \\ ${ }^{2}$ CERN, CH 1211 Geneva 23, Switzerland
}

(Dated: August 6, 2018)

\begin{abstract}
The High Luminosity LHC (HL-LHC) experiments will operate at unprecedented levels of event pile-up from proton-proton collisions at $14 \mathrm{TeV}$ centre-of-mass energy. In this paper, we study the performance of the baseline and a series of alternative scenarios in terms of the delivered integrated luminosity and its quality (pile-up density). A new figure-of-merit is introduced, the effective pileup density, a concept that reflects the expected detector efficiency in the reconstruction of event vertices for a given operational scenario, acting as a link between the machine and experimental sides. Alternative scenarios have been proposed either to improve the baseline performance or to provide operational schemes in the case of particular limitations. Simulations of the evolution of their optimum fills with the latest set of parameters of the HL-LHC are performed with $\beta^{*}$ levelling, and the results are discussed in terms of both the integrated luminosity and the effective pile-up density. The crab kissing scheme, a proposed scenario for pile-up density control, is reevaluated under this new perspective with updated beam and optics parameters. Estimates on the impact of crab cavity noise, full crab crossing, or reduced cross-section for burn-off, on the expected integrated luminosity, are also presented.

PACS numbers: 29.27.-a, 41.58.-p
\end{abstract}

Keywords: Effective pile-up density, Luminosity, Integrated luminosity, HL-LHC, High Luminosity LHC

\footnotetext{
*lmedinam@cern.ch; medinamluis@fisica.ugto.mx
} 


\section{INTRODUCTION}

The High Luminosity LHC (HL-LHC), the luminosity upgrade of the LHC, aims at the increase of the integrated luminosity to about $250 \mathrm{fb}^{-1}$ per year, enabling a ten-fold increase of the integrated luminosity of the LHC over its operational lifetime [1-3]. In order to reach this goal, new beam parameters, accelerator components, and operation techniques have to be implemented [4]: a larger bunch population, reduced beam sizes at the two main interaction points (IPs), the use of crab cavities (CCs), and luminosity levelling with $\beta^{*}$ -tested during the 2018 LHC run $[5,6]-$, are among those of interest for this work.

Since the original HL-LHC proposal [7], the baseline parameters have evolved. In addition, a series of alternative scenarios aiming at improving the potential performance, reducing risks, or providing options in the event of serious limitations, have been studied. For example, in order to minimize the electron cloud effects that could limit the performance of the machine [8], two configurations have been proposed $[9,10]$, namely, the $8 b+4 \mathrm{e}$ filling scheme, and a configuration with a new $200 \mathrm{MHz}$ radiofrequency (RF) system [11]. The use of flat optics, yet to be demonstrated in operation, is adopted in the latter case as a means to minimize performance loss. The impact from the possible absence of crab cavities is addressed, and further reduction of the beam size and the crossing angle at the IP to improve the integrated performance is currently under study too.

As a result of high luminosity levels, detectors will face the challenge of operating at very high pile-up (the average number of events per bunch crossing). It has been found that not only the pile-up has an impact on the detector efficiency, but also its longitudinal distribution around the IP $[12,13]$. In this context, the crab kissing (CK) scheme was proposed as an operational scenario for the control of the event longitudinal density [14], using the peak pile-up density as figure-of-merit.

An original parameter, the effective pile-up density, is introduced in Section II as a better figure-of-merit to compare the different scenarios in terms of their expected detector performance. In Section III we simulate physics fills for the HL-LHC baseline and the aforementioned alternative scenarios, and we discuss their performance considering the effective pile-up density. At the end of this section, an adapted version of the crab kissing scheme with the current flat optics and $\mathrm{CC}$ voltage (but twice the number of CCs than foreseen in the baseline) is discussed. Section IV evaluates the effect of emittance blow-up induced by 
crab cavity noise on the integrated performance. The following sections explore the impact from a larger crab cavity voltage, which enables to perform bunch collisions with full crab crossing, and from a reduced beam burn-off rate due to a smaller magnitude of the crosssection -corresponding to the inelastic cross-section exclusively-, pushing the limits of the achievable integrated luminosity. General and particular conclusions for each scenario are drawn in Section VII.

\section{EFFECTIVE PILE-UP DENSITY}

The pile-up $\mu$ is the average number of events per bunch crossing, and its magnitude changes over time, as the luminosity evolves throughout the fill,

$$
\mu(t)=\frac{\sigma \mathcal{L}(t)}{f n_{b}}
$$

where $\sigma$ is the proton-proton inelastic cross-section, $\mathcal{L}(t)$ is the instantaneous luminosity at time $t, n_{b}$ is the number of colliding bunches, and $f$ is the revolution frequency. A precise and updated characterization of the pile-up level of the different HL-LHC operational scenarios is highly relevant for the experiments: reconstruction and identification of the primary vertices of the events in a collision is a critical first step in most analyses, and their ratio with respect to the number of simulated vertices depends on the pile-up.

A simplified expression of the instantaneous luminosity formula assuming Gaussian distributions for the transversal bunch profiles, the presence of the hour-glass effect, crab crossing in the crossing plane $(\times)$, and crab kissing in the parallel separation plane $(\|)$, is $[14]$

$$
\mathcal{L}=\frac{2 f n_{b} N^{2}}{4 \pi \sigma_{\times}^{*} \sigma_{\|}^{*}} \cos \frac{\theta}{2} \iint \rho_{s}(s-z) \rho_{s}(s+z) K(s, z) \mathrm{d} s \mathrm{~d} z,
$$

where $K(s, z)$ is given by

$$
K(s, z)=\frac{\exp \left(-\frac{\left[\frac{\theta}{2} s-\frac{1}{k_{\times}} \frac{\theta_{\times}}{2} \sin \left(k_{\times} s\right) \cos \left(k_{\times} z\right)\right]^{2}}{\sigma_{\times}^{* 2}\left[1+\left(s / \beta_{\times}^{*}\right)^{2}\right]}-\frac{\left[\frac{1}{k_{\|}} \frac{\theta_{\|}}{2} \cos \left(k_{\|} s\right) \sin \left(k_{\|} z\right)\right]^{2}}{\sigma_{\|}^{* 2}\left[1+\left(s / \beta_{\|}^{*}\right)^{2}\right]}\right)}{\sqrt{1+\left(s / \beta_{\times}^{*}\right)^{2}} \sqrt{1+\left(s / \beta_{\|}^{*}\right)^{2}}},
$$

and $N$ is the bunch population (assumed to be the same for all bunches in both beams), $\theta$ is the full crossing angle, and $\rho_{s}$ is the particle longitudinal density of the two colliding bunches, $\beta_{\times, \|}^{*}$ and $\sigma_{\times, \|}^{*}=\sqrt{\epsilon_{\times, \|} \beta_{\times, \|}^{*}}$ are the transverse betatron functions and beam sizes at the IP, 
and $\epsilon_{\times, \|}$are the emittances in the crossing and separation planes, respectively. The RF crab cavities are characterized by their angles $\theta_{\times, \|}$and wave numbers $k_{\times, \|}=2 \pi f_{\times, \|} / c$, being $f_{\times, \|}$ their frequency. The integrals on the longitudinal coordinate $s$, and on the distance to the reference particle $z$, extend over their respective domains; the IP is located at $s=0$.

The line pile-up density $\rho(s, t)$, or simply pile-up density, describes the local distribution of events along $s$, thus

$$
\mu(t)=\int \rho(s, t) \mathrm{d} s
$$

Note from Eqs. (1) and (4) that $(\mathrm{d} \mathcal{L} / \mathrm{d} s)(s, t)=f n_{b} \rho(s, t) / \sigma$. Experimental tracking detectors are sensitive to the line pile-up density. Other related quantities of interest are the peak (line) pile-up density, usually located at the IP,

$$
\hat{\rho}(t)=\rho(s=0, t)
$$

and the rms luminous region

$$
\sigma_{s, \operatorname{lum}}(t)=\frac{1}{\mu(t)} \int s^{2} \rho(s, t) \mathrm{d} s
$$

both evolving as the fill progresses. Each operational scenario delivers a local density (and luminous region) with particular characteristics. As in the case of the pile-up, a detailed study of these quantities is needed in order to provide the input for accurate simulations of the detector performance for different detector configurations.

The longitudinal resolution of the tracker (around $0.1 \mathrm{~mm}$ ) is significantly smaller than the average distance between pile-up vertices [15], so it is reasonable to assume the impact of pileup density on the detector efficiency to be linear in the current range of collision scenarios. Several quantities have been preliminarily studied as a function of the pile-up density; it has been found that the local efficiency of reconstructed events exhibits an approximately linear relation with the pile-up density $[12,13,16]$, confirming this simple but novel hypothesis. The linear assumption of the effect of the pile-up density on detector efficiency makes it possible to define an effective pile-up density -as derived in the following-, to quantify its integrated effect which is much more interesting than the peak: this figure-of-merit mirrors the integrated luminosity, as opposed to the peak luminosity, as a parameter of interest for the experiments [17].

Based on the results described above, we parametrize the useful data quantity for analysis, represented by $\mathcal{L}_{\text {int }}^{\prime}$, as a fraction of the delivered integrated luminosity $\mathcal{L}_{\text {int }}=\int_{0}^{t_{\text {fill }}} \mathcal{L}(t) \mathrm{d} t$ 
for a fill duration $t_{\text {fill }}$, via a function $f$ of the pile-up $\mu$ and the local density $\rho$,

$$
\mathcal{L}_{\text {int }}^{\prime}=\int_{0}^{t_{\text {fill }}} \int f(\mu, \rho) \mathcal{L}(s, t) \mathrm{d} s \mathrm{~d} t .
$$

Expanding $f$ around $\rho=0$,

$$
f(\mu, \rho) \approx f(\mu, 0)+\left.\frac{\partial f(\mu, \rho)}{\partial \rho}\right|_{\rho=0} \rho(s, t) .
$$

At constant pile-up $\mu_{0}$, which is the case in the HL-LHC for most of the fill duration due to luminosity levelling (a process described in Section III A), the integrals over the first term can be approximated as

$$
\int_{0}^{t_{\text {fill }}} \int f(\mu(t), 0) \mathcal{L}(s, t) \mathrm{d} s \mathrm{~d} t \approx f\left(\mu_{0}\right) \mathcal{L}_{\text {int }},
$$

and

$$
\begin{aligned}
\left.\int_{0}^{t_{\text {fill }}} \int \frac{\partial f\left(\mu_{0}, \rho\right)}{\partial \rho}\right|_{\rho=0} \rho(s, t) \mathcal{L}(s, t) \mathrm{d} s \mathrm{~d} t & \\
& \approx-\varepsilon \int_{0}^{t_{\text {fill }}} \int \rho(s, t) \mathcal{L}(s, t) \mathrm{d} s \mathrm{~d} t \equiv-\varepsilon \bar{\rho} \mathcal{L}_{\text {int }}
\end{aligned}
$$

where

$$
\varepsilon \equiv-\left.\frac{\partial f\left(\mu_{0}, \rho\right)}{\partial \rho}\right|_{\rho=0}
$$

We can then rewrite the useful integrated luminosity in Eq. (7) as

$$
\mathcal{L}_{\text {int }}^{\prime} \approx\left[f\left(\mu_{0}\right)-\varepsilon\left(\mu_{0}\right) \bar{\rho}\right] \mathcal{L}_{\text {int }}, \quad 0 \leq f\left(\mu_{0}\right) \leq 1, \quad \varepsilon>0
$$

and therefore it allows us to compare different scenarios in terms of the integrated luminosity and $\bar{\rho}$ for constant pile-up $\mu_{0}$. Equation (10) defines the effective (line) pile-up density $\bar{\rho}$, the average of the pile-up density over the fill, weighted by the integrated luminosity (or equivalently, the total pile-up in the fill) [18],

$$
\bar{\rho}=\frac{\int_{0}^{t_{\text {fill }}} \int \rho^{2}(s, t) \mathrm{d} s \mathrm{~d} t}{\int_{0}^{t_{\text {fill }}} \mu(t) \mathrm{d} t} .
$$

The units of $\bar{\rho}$ are $\mathrm{mm}^{-1}$, understood as events $/ \mathrm{mm}$. The lower the effective pile-up density is, the higher the detector efficiency becomes for the reconstruction of event vertices, i.e. higher data quality. It has to be noted that for other experimental quantities, such as those not relying on the tracker, only the pile-up level is relevant. 


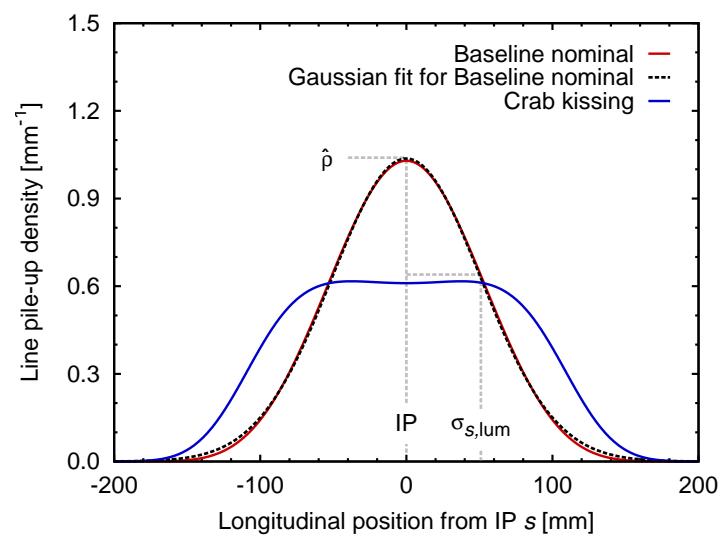

Fig. 1: (Colour online) Pile-up density at IP1 or IP5 at the start of the fill for the HL-LHC nominal baseline (solid red line) and the corresponding Gaussian fit (dotted black line). The original crab kissing scenario (solid blue line) exhibits a flat behaviour at the start of the fill.

\section{A. Approximations}

In general, the pile-up density is a complicated function of the beam and optics parameters at the IP. However, a Gaussian description with peak $\hat{\rho}(t)$ and rms value given by $\sigma_{s, \operatorname{lum}}(t)$,

$$
\rho(s, t)=\hat{\rho}(t) \mathrm{e}^{-s^{2} / 2 \sigma_{s, \mathrm{lum}}^{2}(t)},
$$

is often a good approximation even when considering realistic simulations [19, 20], as seen in Fig. 1. In this case, Eq. (13) can be simplified to

$$
\bar{\rho}_{\text {Gauss }}=\frac{1}{\sqrt{2}} \frac{\int_{0}^{t_{\text {fill }}} \hat{\rho}^{2}(t) \mathrm{d} t}{\int_{0}^{t_{\text {fill }}} \hat{\rho}(t) \mathrm{d} t} .
$$

A flatter event distribution is observed, for example, in the crab kissing scheme at the start of the levelling (Fig. 1). In order to analytically derive the effective pile-up, we consider the extreme case of a rectangular (box) function with full width $\Delta(t)$,

$$
\rho(s, t)=\hat{\rho}(t) \operatorname{box}[s, \Delta(t)]
$$

the effective pile-up density is then given by

$$
\bar{\rho}_{\text {flat }}=\frac{\int_{0}^{t_{\text {fill }}} \hat{\rho}^{2}(t) \mathrm{d} t}{\int_{0}^{t_{\text {fill }}} \hat{\rho}(t) \mathrm{d} t} .
$$

Comparing Eqs. (15) and (17) we observe that $\hat{\rho}$ is not a robust figure-of-merit and it is too pessimistic by about a factor of $\sqrt{2}$ for the cases with approximately Gaussian event 
distributions, such as the HL-LHC baseline. For this reason, we have introduced $\bar{\rho}$, to replace the peak pile-up density as a figure-of-merit to evaluate scenarios in terms of the expected detector performance at constant pile-up.

\section{SIMULATIONS}

\section{A. Luminosity levelling and general settings}

Luminosity decays over time due to beam burn-off and emittance blow-up. In the HLLHC baseline, two levelled luminosities $\left(\mathcal{L}_{\text {lev }}\right)$ at IP1 and IP5 -corresponding to the location of the ATLAS and CMS detectors, respectively- define the nominal (at $5.0 \times 10^{34} \mathrm{~cm}^{-2} \mathrm{~s}^{-1}$ ) and ultimate (at $7.5 \times 10^{34} \mathrm{~cm}^{-2} \mathrm{~s}^{-1}$ ) operation scenarios. Levelling is achieved via stepwise reduction of $\beta^{*}$ at the two main IPs, delivering the desired beam size and crossing angles that provide the above levelled values. At IP8, $\mathcal{L}_{\text {lev }}=2 \times 10^{33} \mathrm{~cm}^{-2} \mathrm{~s}^{-1}$ is assumed to remain constant for the entire fill duration (by levelling with offset, for example). The time evolution of the luminosity is assumed to follow the models in [21, 22] at constant emittance for the duration of each step. A new step, i.e. reduction of $\beta^{*}$ and beam separation, has to be performed once the luminosity has naturally decayed from its initial levelled value of $\mathcal{L}\left(t_{0}\right)=\mathcal{L}_{\text {lev }}$ at the beginning of the step $\left(t=t_{0}\right)$ to a fraction $p$ of it, given by

$$
\mathcal{L}\left(t_{0}+\Delta t_{p}\right)=p \mathcal{L}_{\mathrm{lev}}, \quad \text { for } 0<p<1
$$

after a time $\Delta t_{p}$ given by

$$
\Delta t_{p}=\frac{N_{0}}{r_{0}}\left(\frac{1}{\sqrt{p}}-1\right),
$$

with $N_{0}$ and $r_{0}$ the bunch population and the rate of intensity burn-off, respectively, at time $t_{0}$, and where constant transverse and longitudinal emittance have been assumed for the duration of the step. This levelling process to restore the luminosity by adjusting the optics settings is repeated until the minimum achievable values of $\beta^{*}$ and separation are reached.

The rate of burn-off is pessimistically approximated by the sum of pile-up events in all the IPs, with a total cross-section $\sigma_{\text {b.o. }}$ of $111 \mathrm{mb}[3,23]$,

$$
r(t) \equiv \sum_{\mathrm{IP}} \frac{\sigma_{\text {b.o. }} \mathcal{L}_{\mathrm{IP}}(t)}{n_{b, \mathrm{IP}}}
$$

As the fill progresses -and the bunch population decreases-, the step length $\Delta t_{p}$ shortens. A luminosity step of $2 \%(p=0.98)$ is adopted for the present simulations, and the effect 


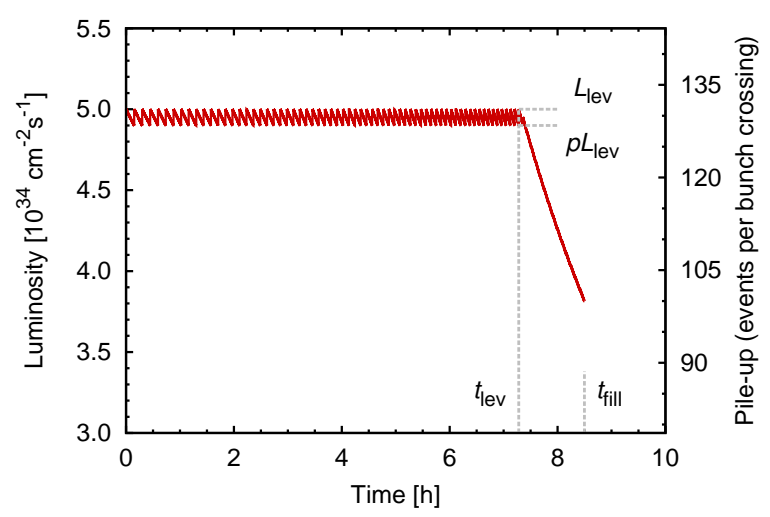

Fig. 2: (Colour online) Evolution of the instantaneous luminosity and pile-up at IP1 or IP5 along the fill for the HL-LHC baseline at nominal levelling.

TABLE I: HL-LHC parameters for different operational scenarios; optics parameters apply to IP1 and IP5.

\begin{tabular}{|c|c|c|c|c|c|c|c|}
\hline Parameter & Unit & Baseline & Flat & $8 \mathrm{~b}+4 \mathrm{e}$ & $200 \mathrm{MHz}$ & No CCs & CK \\
\hline Number of bunches & 1 & 2760 & 2760 & 1972 & 2760 & 2760 & 2760 \\
\hline Number of collisions at IP 1 or IP 5 & 1 & 2748 & 2748 & 1967 & 2748 & 2748 & 2748 \\
\hline Bunch population (protons per bunch, ppb) & $10^{11}$ & 2.2 & 2.2 & 2.2 & 2.2 & 2.2 & 2.2 \\
\hline Total beam current & $\mathrm{A}$ & 1.10 & 1.10 & 0.79 & 1.10 & 1.10 & 1.10 \\
\hline Longitudinal profile & - & q-Gaussian & -Gaussiar & q-Gaussian & Gaussian & q-Gaussian & Supergauss \\
\hline rms bunch length & $\mathrm{cm}$ & 7.6 & 7.6 & 7.6 & 15.0 & 7.6 & 10.0 \\
\hline Full width at half maximum & $\mathrm{cm}$ & 21.2 & 21.2 & 21.2 & 35.3 & 21.2 & 31.4 \\
\hline Relative energy spread & $10^{-4}$ & 1.29 & 1.29 & 1.29 & 1.00 & 1.29 & 1.29 \\
\hline Minimum $\beta_{\times}^{*}, \beta_{\|}^{*}$ & $\mathrm{~cm}$ & $15.0,15.0$ & $18.0,7.5$ & $15.0,15.0$ & $18.0,7.5$ & $31.5,7.5$ & $18.0,7.5$ \\
\hline Full crossing angle & prad & 500 & 490 & 470 & 490 & 410 & 490 \\
\hline Minimum normalized BBLR separation & $\sigma$ & 10.5 & 11.4 & 10.5 & 11.4 & 12.6 & 11.4 \\
\hline Norm. transversal emittance (start) & $\mu \mathrm{m}$ & 2.5 & 2.5 & 2.2 & 2.5 & 2.5 & 2.5 \\
\hline
\end{tabular}

of levelling can be seen in Fig. 2 which shows the luminosity and pile-up for the nominal baseline. Pile-up is estimated with an inelastic proton cross-section of $81 \mathrm{mb}$. Emittance evolution includes radiation damping and intrabeam scattering (IBS) [24], as computed by MAD-X [25]. No additional source of vertical emittance growth, as in [26, 27] is added. The beam energy is $7 \mathrm{TeV}$ for all scenarios.

Table I lists the optics and beam parameters of each operational scenario. The evolution of typical fills for any scenario is simulated by a PYTHON routine, based on the original code implemented in [9], and that has been extended to include new levelling techniques, and to 
better reproduce the latest HL-LHC parameters [28, 29]. The value of $\beta^{*}$ at the beginning of the levelling process is determined by $\mathcal{L}_{\text {lev }}$, taken as the maximum instantaneous luminosity accepted by the detector.

The total fill duration $t_{\text {fill }}$ of each scenario is such that it maximizes the yearly integrated luminosity for each particular case, i.e. the optimum fill. For the estimation of the yearly integrated luminosity, a year accounts for 160 days of operation, with a $50 \%$ performance efficiency -as defined in [30]-, and turn-around times - that is, the time between two consecutive fills- of $145 \mathrm{~min}$ and $150 \mathrm{~min}$ for nominal and ultimate operations, respectively. The expected performances for each scenario are discussed in the following sections.

\section{B. HL-LHC Baseline}

The HL-LHC baseline makes use of round optics with minimum $\beta^{*}=15 \mathrm{~cm}$ and a beam crossing angle of $500 \mu \mathrm{rad}(10.5 \sigma)$ at IP1 and IP5. Two $400 \mathrm{MHz}$ RF crab cavities per IP side and per beam (each with a voltage of $3.4 \mathrm{MV}$ ) provide partial compensation of the crossing angle, which accounts for $380 \mu \mathrm{rad}$ [31].

Previous simulations consider a Gaussian distribution [32] for the modelling of the bunch longitudinal density. In this work, this is described by a particular case of the Tsallis q-Gaussian distribution [33, 34]

$$
\lambda(s) \equiv \frac{4 \sqrt{2}}{5 \pi \sigma_{\lambda}}\left(1-\frac{s^{2}}{8 \sigma_{\lambda}^{2}}\right)^{5 / 2}, \quad|s| \leq 2 \sqrt{2} \sigma_{\lambda},
$$

with bunch length given by the rms value $\sigma_{\lambda}$. This is the distribution observed in the LHC and it is justified for the HL-LHC [19]. The bunch length, and therefore the full width at half maximum (FWHM) -a parameter of interest due to its relation with the longitudinal beam stability-, are kept constant along the fill, and their magnitudes are listed in Table I.

\section{Nominal}

The nominal levelled luminosity of $5.0 \times 10^{34} \mathrm{~cm}^{-2} \mathrm{~s}^{-1}$ yields a total pile-up of 131 events per bunch crossing for the HL-LHC baseline, a perfectly acceptable number in view of the detector capabilities. The steps in the levelling process can be seen in Fig. 2, where the levelling time and fill duration are indicated. 


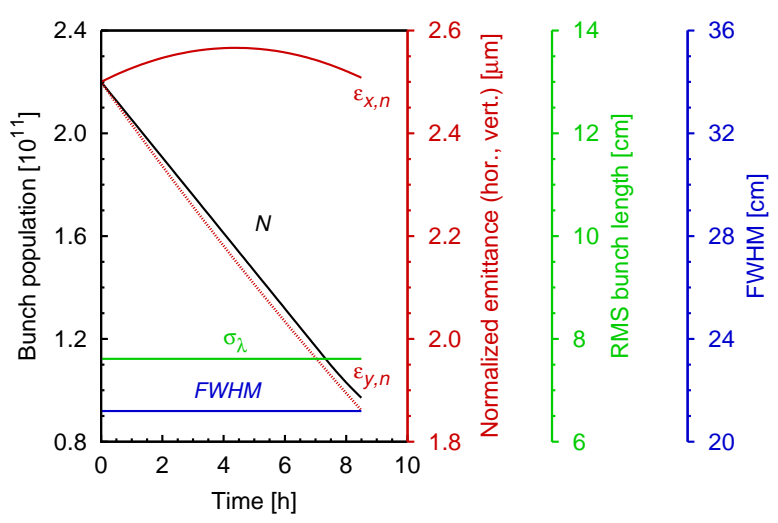

(a)

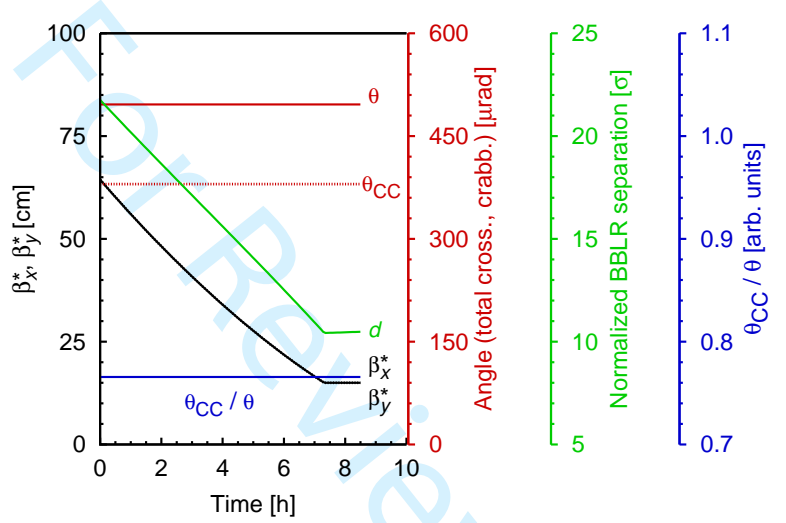

(b)

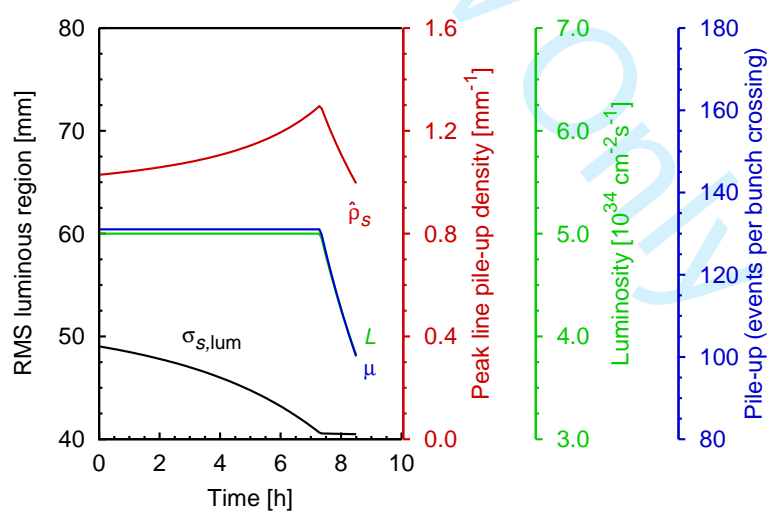

Fig. 3: (Colour online) Evolution of parameters along the fill for the HL-LHC baseline at nominal levelling. (a) Bunch population, normalized emittances, rms bunch length, and FWHM. (b) Normalized BBLR separation, $\beta^{*}$, full crossing angle, crab crossing, and the ratio of the latter two, at IP1 or IP5. (c) rms luminous region, peak line pile-up density, instantaneous luminosity and pile-up, at IP1 or IP5.

In the following, we describe in detail the evolution of the optimum fill for this scenario. 
As seen in Fig. 3(a), the bunch population decays linearly from the beginning of the fill until the end of the levelling. The horizontal emittance reaches a maximum of almost $2.6 \mathrm{\mu m}$ after around $4.5 \mathrm{~h}$; at the end of the fill, it decreases again to approximately its original value. The vertical emittance, on its part, shrinks to about $1.9 \mu \mathrm{m}$ by the end of the fill. At the start of the levelling, $\beta^{*}$ at IP1 or IP5 is $64 \mathrm{~cm}$, as shown in Fig. 3(b). The compensation of the crossing angle by the crab cavity is $\theta_{\mathrm{CC}} / \theta=77 \%$, remaining constant along the fill. The normalized long-range beam-beam (BBLR) separation $d$, for the bunch encounters closer to the IP, decreases from $21.8 \sigma$ to the minimum of $10.5 \sigma$ at the end of the levelling. Sixty-eight steps are needed to keep the luminosity within a $2 \%$-margin from its levelled value; after the minimum $\beta^{*}$ has been reached, the instantaneous decays naturally for $1.2 \mathrm{~h}$ more. A large number of levelling steps might suppose an operational challenge, as each of them represents a different optics for commissioning [35].

The rms luminous region is $49 \mathrm{~mm}$ for the nominal baseline at the start of the fill, and decreases by $17 \%$ towards the end of the fill [see Fig. 3(c)]. The peak pile-up density starts at one event per millimetre; then, due to the reduction of the luminous region, it rises to a maximum $1.30 \mathrm{~mm}^{-1}$ at $t_{\mathrm{lev}}$. After this point, the pile-up density decays as the luminosity naturally decays.

The optimum fill of the HL-LHC nominal baseline is found to deliver a yearly integrated luminosity of $262 \mathrm{fb}^{-1}$-thus, surpassing the current goal-, and the effective pile-up density accounts for $0.80 \mathrm{~mm}^{-1}$. These results are summarized in Table II.

\section{Ultimate}

The ultimate scenario of the HL-LHC exhibits a similar general behaviour than the nominal. However, the levelling time and fill duration are shorter due to the larger levelled luminosity $\left(7.5 \times 10^{35} \mathrm{~cm}^{-2} \mathrm{~s}^{-1}\right)$; this value corresponds to a pile-up of around 200 events per bunch crossing, the maximum value tolerable by the experiments. Regarding the optics, $\beta^{*}$ and the BBLR separation are $41 \mathrm{~cm}$ and $17.3 \sigma$, respectively, at the start of the process; the full crossing angle and crabbing angle, as well as the minimum $\beta^{*}$ and separation remain as in the nominal.

A significant increment is experienced by the pile-up density, which reaches a maximum of $1.95 \mathrm{~mm}^{-1}$ at $t_{\text {lev }}$. As in the nominal operation, the local densities of events are well- 
TABLE II: Results on luminosity, pile-up, and rms luminous region at IP1 or IP5 for the different HL-LHC operational scenarios.

\begin{tabular}{|c|c|c|c|c|c|c|c|c|c|c|c|c|}
\hline \multirow{3}{*}{$\begin{array}{l}\text { Parameter } \\
\text { Levelled luminosity }\end{array}$} & \multirow{3}{*}{$\begin{array}{l}\text { Unit } \\
{ }^{4} \mathrm{~cm}^{-2} \mathrm{~s}^{-1}\end{array}$} & \multirow{2}{*}{\multicolumn{2}{|c|}{$\begin{array}{c}\text { Baseline } \\
\text { Nom. Ult. }\end{array}$}} & \multirow{2}{*}{\multicolumn{2}{|c|}{$\begin{array}{c}\text { Flat } \\
\text { Nom. Ult. }\end{array}$}} & \multirow{2}{*}{\multicolumn{2}{|c|}{$\begin{array}{c}8 \mathrm{~b}+4 \mathrm{e} \\
\text { Nom. Ult. }\end{array}$}} & \multirow{2}{*}{\multicolumn{2}{|c|}{$\begin{array}{c}200 \mathrm{MHz} \\
\text { Nom. Ult. }\end{array}$}} & \multirow{2}{*}{\multicolumn{2}{|c|}{$\begin{array}{c}\text { No CCs } \\
\text { Nom. Ult. }\end{array}$}} & \multirow{3}{*}{$\begin{array}{c}\text { CK } \\
\text { Nom. } \\
5.0\end{array}$} \\
\hline & & & & & & & & & & & & \\
\hline & & 5.0 & 7.5 & 5.0 & 7.5 & 3.8 & 5.5 & 5.0 & 7.5 & 5.0 & 7.5 & \\
\hline Total pile-up & 1 & 131 & 197 & 131 & 197 & 140 & 200 & 131 & 197 & 131 & 197 & 131 \\
\hline Fill duration & $\mathrm{h}$ & 8.5 & 5.3 & 9.1 & 5.7 & 8.2 & 5.5 & 8.1 & 5.2 & 7.4 & 4.9 & 8.9 \\
\hline Levelling time & $\mathrm{h}$ & 7.4 & 3.6 & 8.2 & 4.3 & 7.2 & 4.0 & 6.5 & 2.4 & 5.7 & 2.2 & 8.9 \\
\hline Number of levelling steps & 1 & 68 & 45 & 79 & 56 & 72 & 52 & 57 & 28 & 48 & 25 & 80 \\
\hline \multicolumn{13}{|l|}{ At the start of the fill } \\
\hline Peak pile-up density & $\mathrm{mm}^{-1}$ & 1.03 & 1.62 & 1.00 & 1.56 & 1.07 & 1.58 & 0.82 & 1.48 & 1.23 & 2.13 & 0.61 \\
\hline rms luminous region & $\mathrm{mm}$ & 49 & 47 & 51 & 49 & 50 & 49 & 63 & 54 & 42 & 37 & 67 \\
\hline \multicolumn{13}{|l|}{ At the end of the levelling } \\
\hline Peak pile-up density & $\mathrm{mm}^{-1}$ & 1.30 & 1.95 & 1.34 & 2.02 & 1.31 & 1.88 & 1.29 & 1.93 & 1.81 & 2.73 & 0.61 \\
\hline rms luminous region & $\mathrm{mm}$ & 41 & 41 & 40 & 39 & 43 & 42 & 43 & 44 & 29 & 29 & 58 \\
\hline Effective pile-up density & $\mathrm{mm}^{-1}$ & 0.80 & 1.20 & 0.78 & 1.20 & 0.82 & 1.17 & 0.70 & 1.08 & 1.03 & 1.58 & 0.51 \\
\hline Diff. w.r.t. baseline nominal & $\%$ & & +51 & -2 & +51 & +3 & +47 & -12 & +36 & +29 & +99 & -36 \\
\hline Yearly integrated luminosity & $\mathrm{fb}^{-1} / 160$ days & 262 & 326 & 267 & 340 & 199 & 243 & 256 & 304 & 249 & 293 & 249 \\
\hline Diff. w.r.t. baseline nominal & $\%$ & - & +24 & +2 & +30 & -24 & -7 & -2 & +16 & -5 & +12 & -5 \\
\hline
\end{tabular}

described by Gaussian distributions. In this case, the levelling process involves around 45 steps. The baseline yearly integrated luminosity is $326 \mathrm{fb}^{-1}$-meeting the current goal for this levelling-, which represents an increment of $24 \%$ with respect to the nominal. The effective pile-up density is, in this case, $\bar{\rho}=1.20 \mathrm{~mm}^{-1}$, that is, it rises by around $50 \%$ with respect to the nominal.

\section{Flat optics at the IPs}

This scenario is proposed to feature flat optics with $\beta_{\times}^{*}=18 \mathrm{~cm}$ and $\beta_{\|}^{*}=7.5 \mathrm{~cm}$ at IP1 and IP5, values that maximize the virtual luminosity (the luminosity achievable with the maximum bunch population -corresponding at the beginning of the fill-, and with the minimum $\beta^{*}$-to be reached at the end of the fill-). The levelling is performed keeping the $\beta_{\times}^{*} / \beta_{\|}^{*}$ ratio constant. With a BBLR separation of $11.4 \sigma$-an optimistic assumption that has yet to be demonstrated for flat optics, and that might require the implementation of long-range compensation techniques [36-38]-, the full crossing angle is around $490 \mu \mathrm{rad}$. The fraction of compensation of the crossing angle by the crab cavities is approximately the same 
than the baseline. The Flat alternative, yet to be further validated, pushes the performance by $2 \%$ and $4 \%$ for the nominal and ultimate levelling, respectively, while reducing the effective pile-up density for the nominal by $2 \%$, and preserving its magnitude for ultimate operation. Baseline detector performance $\left(\mathcal{L}_{\text {int }}^{\prime}\right)$ is therefore expected in the Flat alternative scenario.

\section{D. $8 b+4 e$}

The $8 \mathrm{~b}+4 \mathrm{e}$ filling scheme, aiming at addressing potential electron-could limitations, makes use of fewer bunches, the baseline round optics, and smaller emittance (Table I). Luminosity is also levelled at a lower magnitude, namely, $3.8 \times 10^{34} \mathrm{~cm}^{-2} \mathrm{~s}^{-1}$ (nominal) or $5.5 \times 10^{34} \mathrm{~cm}^{-2} \mathrm{~s}^{-1}$ (ultimate), resulting nonetheless in a slightly higher pile-up compared to the HL-LHC baseline, but consistent with the maximum pile-up tolerable by the experiments. Pile-up densities -peak and effective-, are slightly larger (smaller) than those for the baseline for nominal (ultimate) operation, although their magnitude does not differ significantly (less than $3 \%$ ). The $8 \mathrm{~b}+4 \mathrm{e}$ alternative reduces, however, the nominal (ultimate) integrated performance by $24 \%$ (26\%) in comparison with its baseline counterpart. Therefore, from the experimental point of view, detector efficiency is not expected to be significantly affected in the nominal $8 b+4 \mathrm{e}$ alternative but, on the contrary, it is expected to rise by a few percents at ultimate operation. As in the previous cases, the longitudinal density of events is well-described by a Gaussian distribution at any time along the fill.

\section{E. $200 \mathrm{MHz}$ scenario}

Constituting another alternative scenario for the mitigation of electron-cloud effects, the $200 \mathrm{MHz}$ scheme is characterized by a longer bunch length and a secondary RF system (with a total voltage of $6 \mathrm{MV}$ ) [39]. Studies with a Gaussian longitudinal profile and a $15 \mathrm{~cm}-\mathrm{rms}$ bunch length have found mitigation of the heat load for a secondary emission yield of 1.4 [40]. Simulation of the optimum fill assumes that the bunch length decreases due to synchrotron radiation damping. The longitudinal stability of this case is guaranteed by the main LHC RF system operating at $400 \mathrm{MHz}$, which can be used for double RF harmonic operation. No additional space is expected to be required for the new low-harmonic system as it would 
replace two of the existing $400 \mathrm{MHz}$ RF modules. Simulations at zero chromaticity have shown a reduction of the transverse mode couple instability threshold to $2.6 \times 10^{11}$ protons per bunch [41]. This value is above the foreseen operational bunch charge; it is possible, however, that multi-bunch effects slightly decrease the threshold approaching the operational bunch charge [3].

At nominal pile-up level, the $200 \mathrm{MHz}$ alternative -with identical optics to the Flat scenario- delivers one of the largest luminous regions $(63 \mathrm{~mm})$, while reducing the effective pile-up density by $12 \%$. In terms of luminosity performance, little loss is observed with respect to the baseline. Despite the lower $\mathcal{L}_{\text {int }}$, first detector studies find the efficiency in the $200 \mathrm{MHz}$ scenario to be better than the baseline thanks to the lower $\bar{\rho}$. These differences narrow at ultimate operation nonetheless.

\section{F. Absence of crab cavities}

Due to possible initial issues with their operation or delays in their installation, crab cavities could be absent at the HL-LHC. At nominal levelling, the yearly integrated luminosity of the baseline (round optics) is found to be $229 \mathrm{fb}^{-1}$, that is, a reduction of more than $12 \%$. Similarly, the different alternative scenarios discussed in the previous sections, i.e. Flat, $8 \mathrm{~b}+4 \mathrm{e}$ and $200 \mathrm{MHz}$, experience performance losses of $8 \%, 12 \%$, and $17 \%$, respectively when crab cavities are not present (nominal levelling). Effective pile-up density is affected as well, with the scenarios featuring flat optics delivering the lowest values: $1.38 \mathrm{~mm}^{-1}$ in Flat and $1.46 \mathrm{~mm}^{-1}$ in $200 \mathrm{MHz}$, in comparison with $1.57 \mathrm{~mm}^{-1}$ in the baseline, and $1.63 \mathrm{~mm}^{-1}$ in $8 \mathrm{~b}+4 \mathrm{e}$, values of $\bar{\rho}$ that even surpass those found at their ultimate levelling counterparts with crab cavities. These figures, as well as the impact on the RMS luminous region, fill duration and levelling time, are listed in Table III.

The situation considerably worsens at ultimate operation, where the reduction of integrated luminosity doubles due to the absence of crabbing, and the effective pile-up density rises beyond $2 \mathrm{~mm}^{-1}$ in all cases (except the $200 \mathrm{MHz}$ scenario, where levelling at $\mathcal{L}_{\text {lev }}=7.5 \times 10^{34} \mathrm{~cm}^{-2} \mathrm{~s}^{-1}$ is not reachable without crab cavities for its current optics).

Hence, in the event of no crab cavities, the use of flat optics is mandatory. In particular, $\beta_{\times}^{*} / \beta_{\|}^{*}=31.7 \mathrm{~cm} / 7.5 \mathrm{~cm}$ and a $12.6 \sigma$-normalized BBLR separation is found to maximize the virtual luminosity. This scenario - that has yet to be validated, as the previous scenarios 
TABLE III: Impact of the absence of crab cavities on the luminosity, pile-up, and rms luminous region at IP1 or IP5 for the selected HL-LHC operational scenarios.

\begin{tabular}{|c|c|c|c|c|c|c|c|c|}
\hline \multirow{3}{*}{$\begin{array}{l}\text { Parameter } \\
\text { Fill duration }\end{array}$} & \multirow{3}{*}{$\frac{\text { Unit }}{\mathrm{h}}$} & \multirow{2}{*}{\multicolumn{2}{|c|}{$\begin{array}{c}\text { Baseline } \\
\text { Nom. Ult. }\end{array}$}} & \multirow{2}{*}{\multicolumn{2}{|c|}{$\begin{array}{c}\text { Flat } \\
\text { Nom. Ult. }\end{array}$}} & \multirow{2}{*}{\multicolumn{2}{|c|}{$\begin{array}{c}8 \mathrm{~b}+4 \mathrm{e} \\
\text { Nom. Ult. }\end{array}$}} & \multirow{3}{*}{$\begin{array}{c}200 \mathrm{MHz} \\
\text { Nom. } \\
6.5\end{array}$} \\
\hline & & & & & & & & \\
\hline & & 6.4 & 5.1 & 7.2 & 4.8 & 6.2 & 4.8 & \\
\hline Levelling time & $\mathrm{h}$ & 3.6 & 0.5 & 5.4 & 1.9 & 3.7 & 1.0 & 2.1 \\
\hline Number of levelling steps & 1 & 28 & 6 & 45 & 22 & 31 & 12 & 16 \\
\hline At the start of the fill & & & & & & & & \\
\hline Peak pile-up density & $\mathrm{mm}^{-1}$ & 1.94 & 3.85 & 1.53 & 2.88 & 1.99 & 3.61 & 2.02 \\
\hline RMS luminous region & $\mathrm{mm}$ & 27 & 21 & 34 & 27 & 28 & 22 & 26 \\
\hline At the end of the levelling & & & & & & & & \\
\hline Peak pile-up density & $\mathrm{mm}^{-1}$ & 2.66 & 4.07 & 2.51 & 3.79 & 2.80 & 4.12 & 2.48 \\
\hline RMS luminous region & $\mathrm{mm}$ & 20 & 20 & 21 & 21 & 20 & 20 & 22 \\
\hline Effective pile-up density & $\mathrm{mm}^{-1}$ & 1.57 & 2.13 & 1.38 & 2.17 & 1.63 & 2.25 & 1.46 \\
\hline Diff. w.r.t. baseline nominal & $\%$ & - & +36 & -12 & +39 & +4 & +44 & -7 \\
\hline Yearly integrated luminosity & $\mathrm{fb}^{-1} / 160$ days & 229 & 248 & 247 & 288 & 174 & 190 & 214 \\
\hline Diff. w.r.t. baseline nominal & $\%$ & - & +8 & +8 & +26 & -24 & -17 & -7 \\
\hline
\end{tabular}

with flat beams-, delivers the figures listed in Table II: notoriously, the loss on integrated luminosity is only $5 \%(10 \%)$ at nominal (ultimate) operation, but it comes with a larger effective pile-up (about $1.0 \mathrm{~mm}^{-1}$ for nominal and $1.6 \mathrm{~mm}^{-1}$ for ultimate). Limiting the maximum peak pile-up density, and $\bar{\rho}$ consequently, can be implemented as part of the levelling (it is accomplished by slowing down the rate of the reduction of $\beta^{*}$ ), but it comes at a greater cost on the integrated luminosity, as seen in Fig. 4. The balance between these two parameters is currently being evaluated by the experiments.

\section{G. Crab kissing scheme}

In the original crab kissing concept [14], the process of levelling not only keeps the luminosity under control, but a maximum value on the peak pile-up density is also enforced -similar to the limit discussed at the end of the previous section-. In the operation with crab kissing, however, this is accomplished by the use of two knobs, the crab crossing angle (in the crossing plane) and the crab kissing angle (in the parallel separation plane). The $\beta$ functions at the IP are kept at their minimum values for the entire fill. The crab kissing angle is first adjusted to achieve the desired peak pile-up density; this, in turn, reduces the 


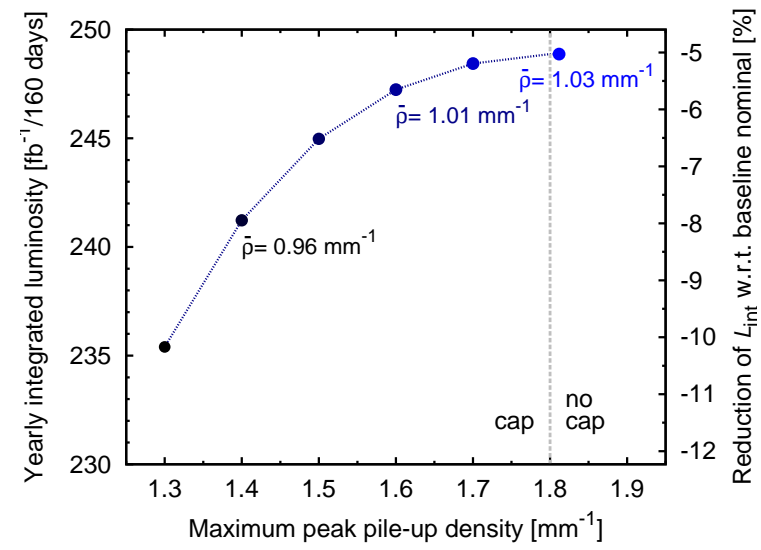

Fig. 4: (Colour online) Integrated luminosity as a function of the maximum peak pile-up density for the case without crab cavities (flat optics). Only the case with the largest peak pile-up density is simulated without constraint in its magnitude; in all other cases, the maximum peak pile-up densities correspond to the applied cap for each of them. Effective pile-up densities are shown for selected points.

luminosity, which is then levelled by adjusting the crab crossing angle. Once the crossing angle is fully compensated by the crab cavities in the crossing plane, and the kissing angle has reduced to zero, the luminosity decays naturally. This results in three stages throughout the fill. Operation with crab kissing thus requires the use of four crab cavities per beam and per IP side, to deflect the bunches in both the transversal planes, making it incompatible with the current HL-LHC baseline.

The CK scheme remains of general interest as it delivers the lowest effective pile-up density (which translates into the largest detector efficiency), and its reduction of integrated luminosity with respect to the baseline can be small under certain conditions. The crab kissing scheme has been reproduced with identical machine and beam parameters to those in [14] with the aim to characterize it in terms of both the integrated performance and the expected detector efficiency (via de newly introduced figure-of-merit), in a similar way to all the current alternative scenarios presented here; additionally, this allows us also to validate the simulation tools. The integrated luminosity for an optimum fill is not discussed in [14], where the very important role of turn-around time is omitted. For the original CK, the optimum fill duration is found to be $8.8 \mathrm{~h}$ (nominal turn-around time is assumed), with an effective pile-up density of $0.50 \mathrm{~mm}^{-1}$ for a constant peak pile-up density of $0.61 \mathrm{~mm}^{-1}$ during the entire fill duration; the yearly integrated luminosity is $245 \mathrm{fb}^{-1} / 160$ days, that is, 


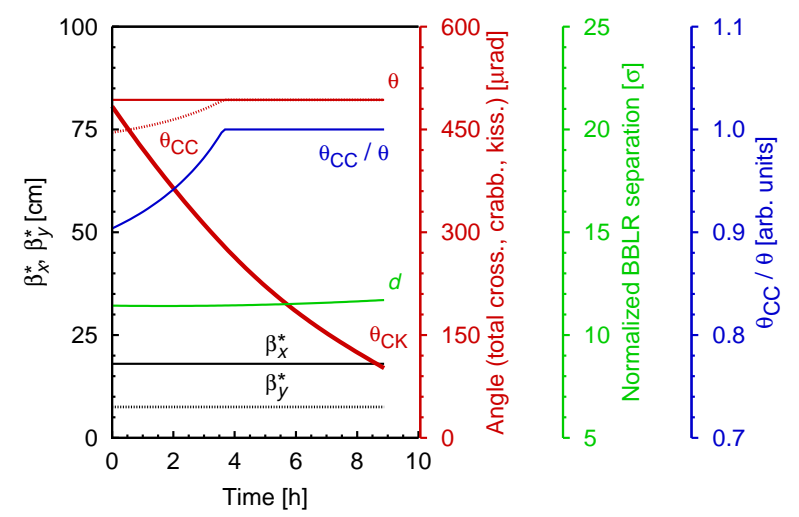

(a)

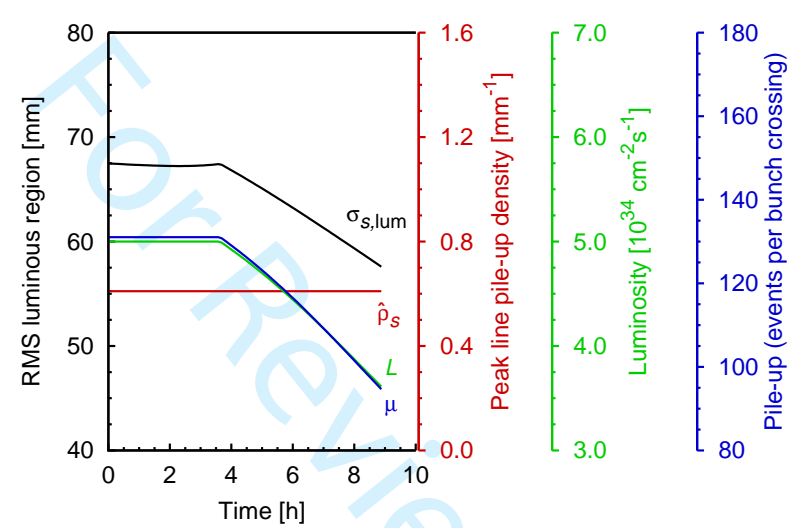

(b)

Fig. 5: (Colour online) Evolution of parameters along the fill for the crab kissing scheme at nominal levelling. (a) Normalized BBLR separation, $\beta^{*}$, full crossing angle, and crab kissing, at IP1 or IP5. (b) rms luminous region, peak line pile-up density, instantaneous luminosity and pile-up, at IP1 or IP5.

a loss of more than $9 \%$ with respect to the original HL-LHC described in the aforementioned reference under the same conditions.

Operation with crab kissing has been re-evaluated with the same flat optics from Section III C and revised CC voltage (see Table I). As in the original CK scheme, the longitudinal density is described by a supergaussian function of order 4 [42],

$$
\nu(s)=\frac{2^{3 / 5}}{\Gamma(1 / 4) S_{\nu}} \exp \left(-\frac{s^{4}}{2 S_{\nu}^{4}}\right),
$$

with rms

$$
\sigma_{\nu} \equiv S_{\nu}\left(\sqrt{2} \frac{\Gamma(3 / 4)}{\Gamma(1 / 4)}\right)^{1 / 2}=10 \mathrm{~cm} .
$$

At the beginning of the fill, bunches are crabbed with $\theta_{\mathrm{CC}} / \theta=90 \%$ [see Fig. $5(\mathrm{a})$ ] and, 


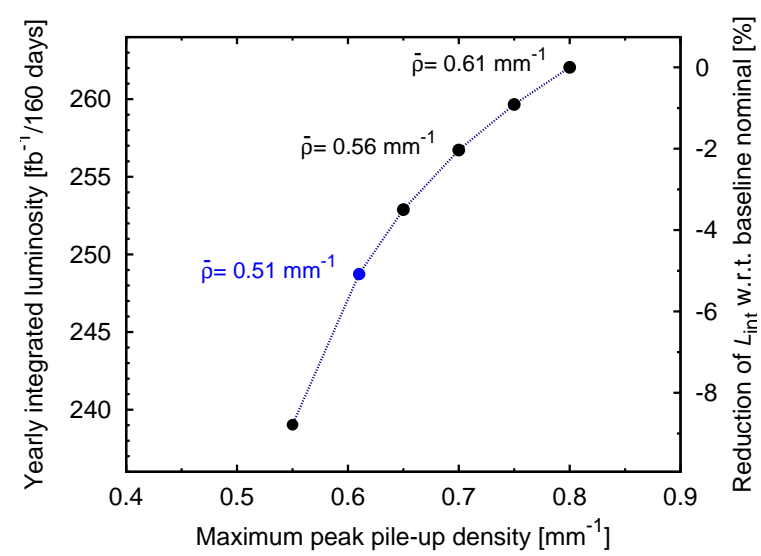

Fig. 6: (Colour online) Integrated luminosity as a function of the maximum peak pile-up density for the crab kissing scheme. Effective pile-up densities shown for selected points.

after $4 \mathrm{~h}$, the 490 urad crossing angle is fully compensated. Parallelly, a crab kissing angle of almost the same magnitude than the crossing angle lowers the peak pile-up density to $0.61 \mathrm{~mm}^{-1}$, that is, less than half of the nominal baseline; a value that is kept constant over the entire the fill. During this first stage of levelling, a constant pile-up of 131 events per bunch, and an rms luminous region of around $68 \mathrm{~mm}$ are maintained.

For the CK scenario with the current flat optics, the optimum fill duration is found to be $8.9 \mathrm{~h}$, and a zero-crab kissing angle is not reached at the end of fill. During the second stage, the luminosity and pile up drop almost linearly, reaching $3.6 \times 10^{34} \mathrm{~cm}^{-2} \mathrm{~s}^{-1}$ and 95 events per bunch crossing, respectively. This updated CK scheme delivers a yearly integrated luminosity of $249 \mathrm{fb}^{-1}$, representing a $5 \%$-reduction with respect to the current HL-LHC nominal baseline. The effective line pile-up density is the lowest among the studied scenarios, $0.51 \mathrm{~mm}^{-1}$ (Table II). It has to be highlighted again, nevertheless, the need of doubling the number of crab cavities to run the machine under this alternative operation.

The maximum peak pile-up density -and the effective pile-up in consequence- can be pushed to even smaller values; doing this, nonetheless, can lead to important loss of integrated performance (for example, $9 \%$ at $\bar{\rho}=0.47 \mathrm{~mm}^{-1}$ ). Contrary, by relaxing the constraint on $\hat{\rho}$ it is possible to almost fully recover the nominal baseline integrated luminosity, but lower detector efficiency is expected, as shown in Fig. 6. As in the case of the absence of crab cavities, the limit in the maximum peak pile-up density is set by the experiments. 


\section{IMPACT OF CRAB CAVITY NOISE}

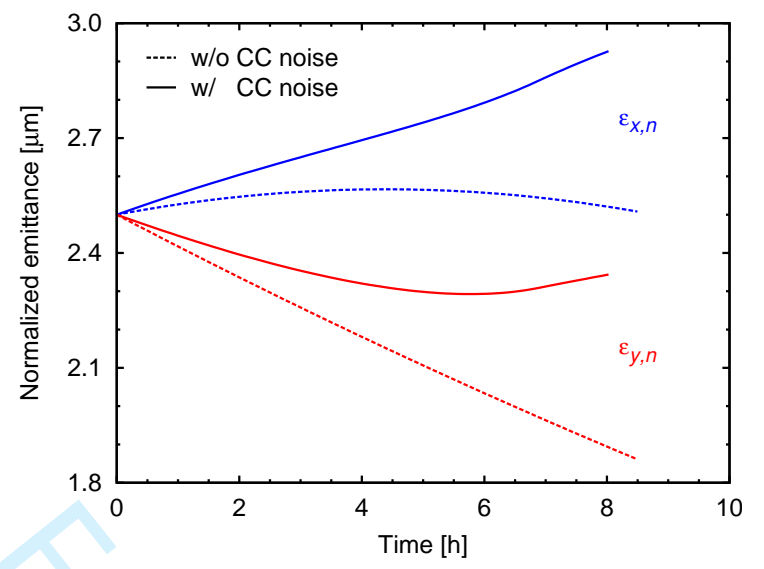

Fig. 7: (Colour online) Evolution of normalized emittances at IP1 or IP5 along the fill for the HL-LHC baseline at nominal levelling with and without crab cavity noise $(4.6 \% / \mathrm{h}$ for $\beta^{*}=15 \mathrm{~cm}$ and $\left.6.8 \mathrm{MV}\right)$.

Crab cavity noise is an important concern since it can generate emittance blow-up [43, 44]. Preliminary simulations have been conducted to asses the effect of the emittance growth from crab cavities, approximated by

$$
\left(\frac{d \epsilon}{d t}\right)_{\mathrm{CC}} \approx 0.115 \mu \mathrm{m} / \mathrm{h} \cdot \frac{V_{\mathrm{CC}}^{2}}{(6.8 \mathrm{MV})^{2}} \frac{15 \mathrm{~cm}}{\beta^{*}} .
$$

The additive growth rate of $0.115 \mu \mathrm{m} / \mathrm{h}$ is taken from the sum of the phase noise $(0.94 \% / \mathrm{h})$ and amplitude noise $(3.7 \% / \mathrm{h})$ of the $\mathrm{CC}$ voltage, estimated for a transversal emittance of $2.5 \mu \mathrm{m}, \beta^{*}=15 \mathrm{~cm}$, and a $3.4 \mathrm{MV}$-deflecting voltage for each of the two crab cavities per beam and per IP side ( or $\theta_{\mathrm{CC}}=380 \mu \mathrm{rad}$ ), according to present estimates of the minimum noise level achievable, and taking into account the effect of the transverse feedback [45].

Figure 7 illustrates the influence of the crab cavity noise on the evolution of the horizontal and vertical emittances along the fill for the HL-LHC nominal baseline (the effects of IBS and synchrotron radiation, as explained in Section III A, are also present). At the beginning of the fill, the crab cavities around the main IPs induce emittance blow-up in both planes -horizontal (vertical) crossing is assumed in IP1 (IP5) - at a rate of $0.027 \mu \mathrm{m} / \mathrm{h}$ due to larger $\beta^{*}$. At nominal operation, the reduction of $\mathcal{L}_{\text {int }}$ with respect to the case without $\mathrm{CC}$ noise is around $2 \%$, and it exhibits a linear behaviour for smaller growth rates. The Flat alternative experiences only a $1 \%$ luminosity loss, while the reduction for the $8 \mathrm{~b}+4 \mathrm{e}$ and $200 \mathrm{MHz}$ 
TABLE IV: Effective pile-up density and luminosity at IP1 or IP5 for the different HL-LHC operational scenarios assuming a $0.115 \mu \mathrm{m} / \mathrm{h}$ emittance growth from crab cavities (for $\beta^{*}=15 \mathrm{~cm}$ and $6.8 \mathrm{MV}$, and scaled correspondingly).

\begin{tabular}{|c|c|c|c|c|c|c|c|c|c|c|c|c|}
\hline \multirow{2}{*}{ Parameter } & \multirow{2}{*}{ Unit } & \multicolumn{2}{|c|}{ Baseline } & \multicolumn{2}{|c|}{ Flat } & \multicolumn{2}{|c|}{$8 b+4 e$} & \multicolumn{2}{|c|}{$200 \mathrm{MHz}$} & \multicolumn{2}{|c|}{ No CCs } & \multirow{2}{*}{$\frac{\text { CK }}{\text { Nom. }}$} \\
\hline & & Nom. & Ult. & Nom. & Ult. & Nom. & Ult. & Nom. & Ult. & Nom. & Ult. & \\
\hline Yearly integrated luminosity & $\mathrm{fb}^{-1} / 160$ days & 258 & 319 & 265 & 335 & 196 & 237 & 252 & 295 & 249 & 293 & 231 \\
\hline Diff. w.r.t. $0 \mu \mathrm{m} / \mathrm{h}$ & $\%$ & -1.5 & -2.3 & -1.0 & -1.5 & -1.5 & -2.2 & -1.6 & -2.7 & - & - & -7.0 \\
\hline Diff. w.r.t. baseline nominal & $\%$ & - & +23 & +3 & +30 & -24 & -8 & -2 & +15 & -4 & +14 & -10 \\
\hline
\end{tabular}

scenarios are similar to the baseline. At ultimate levelling, these figures increase by around $50 \%$, as listed in Table IV, where the difference of $\mathcal{L}_{\text {int }}$ with respect to the baseline is also included. The impact of CC noise is more dramatic for the crab kissing scheme, due to the use of two additional RF cavities, where the integrated luminosity is reduced by $7 \%$; this represents a performance $10 \%$-lower than the nominal with the same noise rate. The effect of CC noise on the original crab kissing operation is halved due to larger $\beta^{*}$.

Both the fill duration of the optimum fill, as well as the levelling time, are also shortened by the $\mathrm{CC}$ noise, while the change on $\bar{\rho}$ is almost negligible. These preliminary results have triggered efforts to mitigate crab cavity noise at the source, or by introducing a feedback system acting on $\mathrm{CC}$ voltage and phase based on the measurement of the head-tail motion at a dedicated pick-up $[45,46]$.

\section{REDUCED TOTAL CROSS-SECTION FOR BURN-OFF}

It should be noted that simulations have been conducted assuming a pessimistic burn-off with a cross-section of $111 \mathrm{mb}$ (corresponding to the contributions of both elastic and inelastic processes), an empirical estimate based on LHC observations (at lower bunch charge) at the beginning of the fill. Observations also show, however, that the magnitude of the cross-section for burn-off tends to $81 \mathrm{mb}$ with time. Proportional increments between $7 \%$ to $8 \%$, and between $11 \%-12 \%$ on integrated luminosity for nominal and ultimate operations, respectively, are expected for each scenario with the more optimistic assumption of beam burn-off given by the inelastic cross-section exclusively $(81 \mathrm{mb})$. The extent of the performance in terms of $\mathcal{L}_{\text {int }}$ and $\bar{\rho}$ for the different scenarios studied here, with the two values of cross-section and two pile-up levels as extreme points, is presented in Fig. 8 (excluding CK 


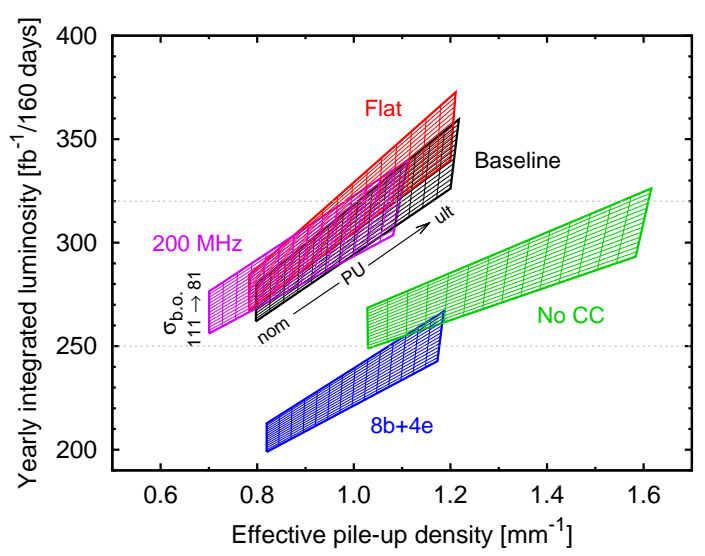

Fig. 8: (Colour online) Extent of the integrated luminosity and effective pile-up density of the different HL-LHC operational scenarios for the cases with $\sigma_{\text {b.o. }}=111 \mathrm{mb}$ and $81 \mathrm{mb}$ and pile-up (PU) levels corresponding to the nominal and ultimate operations of each scenario.

since it is not compatible with the current HL-LHC baseline for needing twice the number of CCs).

\section{FULL CRABBING VOLTAGE}

We explore the potential performance that can be reached with additional CC voltage, coming from four crab cavities per beam per IP side, since it remains of interest for the HL-LHC project as a future upgrade to the machine.

For the baseline optics, in particular, $9.0 \mathrm{MV}$ fully crabs the beam i.e. $\theta_{\mathrm{CC}} / \theta=1$ (see Fig. 9). Despite the gain on integrated performance with respect to the case with 6.4 MV being small (around $2 \%$ for nominal and $3 \%$ for ultimate), the effective pile-up density does experience a moderate improvement: $0.71 \mathrm{~mm}^{-1}$ and $1.06 \mathrm{~mm}^{-1}$, respectively, positively impacting on detector efficiency. With four crab cavities available, the crab crossing angle can be even pushed slightly above the crossing angle, although the resulting gains $\mathcal{L}_{\text {int }}$ and $\bar{\rho}$ in such conditions are negligible.

Full crab crossing for the Flat and $8 \mathrm{~b}+4 \mathrm{e}$ alternatives yields an increase of $1 \%(2 \%)$ at nominal (ultimate) operation on integrated performance, with effective pile-up densities similar to the baseline. The $200 \mathrm{MHz}$ option profits the most from full crabbing, with a $3 \%$-gain on integrated luminosity for nominal -and double this figure for ultimate-, and the remarkably low effective pile-up densities: $0.58 \mathrm{~mm}^{-1}$ and $0.89 \mathrm{~mm}^{-1}$, respectively. 

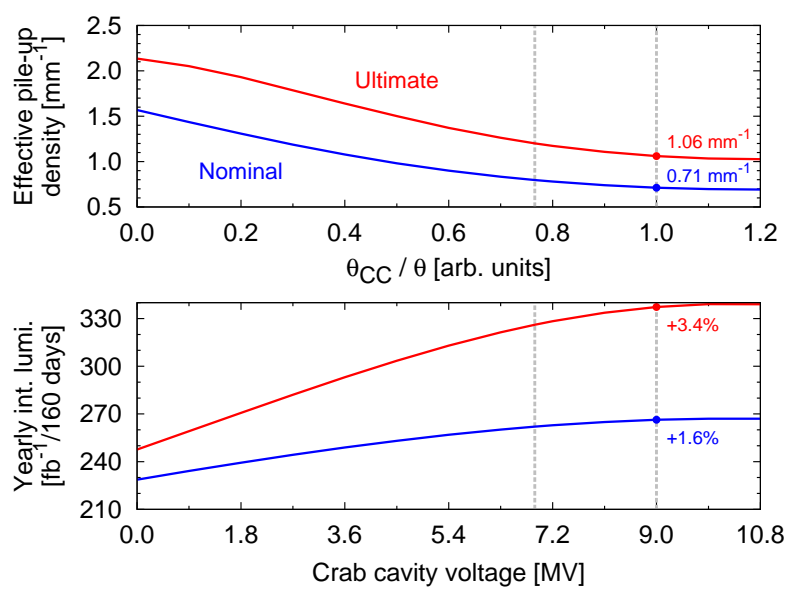

Fig. 9: (Colour online) Effective pile-up density and integrated luminosity as a function of

the crab cavity voltage (or alternatively, of the corresponding fraction of compensated angle by crab crossing. The values of effective pile-up density and integrated luminosity for full crabbing $\left(\theta_{\mathrm{CC}} / \theta=1\right)$ are shown.

Combined full crabbing voltage and reduced cross-section $(81 \mathrm{mb})$ can boost the current estimate on integrated luminosity by $8 \%$ for nominal operation, and by up to $14 \%$ at ultimate pile-up.

\section{CONCLUSIONS}

In view of the unprecedented levels of pile-up and pile-up density that the experiments will face in the HL-LHC upgrade, a detailed characterization of these parameters for the baseline and the different alternative scenarios is extremely important. The concept of a new figure-of-merit, the effective pile-up density $\bar{\rho}$, has been introduced as a means to evaluate the operational scenarios taking into account detector performance. The introduction of this parameter is motivated from detector simulations $[12,13]$ showing a linear relation of the detector efficiency in the reconstruction of primary vertices with the local density -not with the peak pile-up density, commonly considered in the past--, and extrapolating to its average value along the fill. In the same simulations, it has been found that the pile-up density can also affect the proper assignment of non-primary tracks to the primary vertex.

The concept of effective pile-up density is based on the pile-up density introduced in [14], but it extends it to an integrated average over the fill duration, to mirror the integrated 
luminosity as figure-of-merit to characterize the optimum fills of a given operational scenario in the sense that experiments are often more interested on the integrated luminosity, than on its peak at all times. While the peak pile-up density remains of interest, the effective pileup aims at better describing the expected detector performance. In consequence, relative comparisons of performance for different scenarios can be done in terms of their integrated luminosity and effective pile-up densities.

In terms of data quantity, the current baseline delivers around $262 \mathrm{fb}^{-1}$ per year for a pile-up of 131 events per bunch crossing (nominal), and $326 \mathrm{fb}^{-1}$ per year for a pile-up of almost 200 events per bunch crossing (ultimate), not only meeting, but surpassing, the HLLHC goals at each operation, namely $3000 \mathrm{fb}^{-1}$ and $4000 \mathrm{fb}^{-1}$ after 12 years of operation. In terms of data quality, the effective pile-up densities are $0.80 \mathrm{~mm}^{-1}$ (nominal) and $1.20 \mathrm{~mm}^{-1}$ (ultimate).

Regarding the alternative scenarios -which provide extreme cases of study for the experiments-, flat optics, as implemented in the Flat and $200 \mathrm{MHz}$ scenarios, not only restores/preserves the integrated performance, but also leads to slightly reduced effective pile-up densities, from which detector efficiency can profit. In particular, the latter scenario, delivers around $10 \%$ lower $\bar{\rho}$ due to the largest rms luminous regions coming from longer bunches. The use of flat optics in the absence of crab cavities also manages to limit the performance loss below $5 \%$, but comes at the expense of larger effective pile-up densities. It must be noted that flat optics has not been tested yet, and that the implications of this mode of operation should be thoroughly assessed. Baseline detector performance is expected in the $8 \mathrm{~b}+4 \mathrm{e}$ scenario, but it comes with a much larger impact on the integrated luminosity than the other alternatives.

Characterization in terms of the effective pile-up density and yearly integrated luminosity of the crab kissing scheme -reproduced as originally proposed [14]-for the optimum fill, finds $\bar{\rho}=0.51 \mathrm{~mm}^{-1}$, and a reduction of $9 \%$ of $\mathcal{L}_{\text {int }}$; these results are explained by the flatter distribution of the pile-up density at the beginning of the fill. An updated version for this scenario has been presented, making use of similar parameters and simulation settings to those of the current Flat scenario. The integrated luminosity is found to be reduced by $5 \%$ with respect to the current nominal baseline, while delivering the lowest effective pile-up density. It has to be stressed, nonetheless, that four crab cavities per beam and per IP side are needed for the crab kissing operation, as opposed to two in the current HL-LHC baseline. 
It seems then that in order to achieve an important reduction of the effective pile-up density, a major hardware component has to be involved, such as additional crab cavities to perform crab kissing, or a new $200 \mathrm{MHz}$ RF system.

First estimates of the impact of emittance blow up induced by crab cavity noise show reductions by $1 \%$ to $3 \%$ on the integrated luminosity for all scenarios. A notable exception is the crab kissing scheme, whose luminosity performance can be seriously compromised by $\mathrm{CC}$ noise. Therefore implementation of mitigation techniques is of great importance. Availability of full crabbing voltage leads to the increment of the integrated luminosity of the different scenarios by few percent, at the same time that lowers their effective pile-up densities; this translates into improved amounts of data and their quality, that the collisions at each of these operational scenarios can provide.

\section{ACKNOWLEDGEMENTS}

The authors thank B. Petersen, P. Azzi, and the Experimental Data Quality group for the fruitful collaboration that led to the introduction of the concept of effective pile-up density, to S. Fartoukh for valuable discussions regarding the crab kissing scheme, and to O. Brüning, R. Calaga, M. Giovannozzi, G. Iadarola, Y. Papaphilippou, and L. Rossi for their comments throughout the development of this work and the preparation of this manuscript.

[1] The HL-LHC Project, May 2018. URL: http://hilumilhc.web.cern.ch/.

[2] O. Brüning and L. Rossi. The High Luminosity Large Hadron Collider: The New Machine for Illuminating the Mysteries of Universe. World Scientific, 2015.

[3] G. Apollinari, I. Béjar Alonso, O. Brüning, M. Lamont, and L. Rossi, editors. High-Luminosity Large Hadron Collider (HL-LHC) Technical Design Report V0.1. CERN, Geneva, 2017. unpublished. URL: https://edms . cern.ch/document/1833445.

[4] G. Arduini, J. Barranco, A. Bertarelli, N. Biancacci, R. Bruce, O. Brüning, X. Buffat, Y. Cai, L.R. Carver, S. Fartoukh, M. Giovannozzi, G. Iadarola, K. Li, A. Lechner, L. Medina Medrano, E. Métral, Y. Nosochkov, Y. Papaphilippou, D. Pellegrini, T. Pieloni, J. Qiang, S. Redaelli, A. Romano, L. Rossi, G. Rumolo, B. Salvant, M. Schenk, C. Tambasco, R. Tomás, S. Valishev, 
and F.F. Van der Veken. High Luminosity LHC: challenges and plans. Journal of Instrumentation, 11(12):C12081, 2016. URL: http://stacks.iop.org/1748-0221/11/i=12/a=C12081.

[5] J. Wenninger, K. Fuchsberger, M. Hostettler, M. Pojer, L. Ponce, and T. Tydecks. $\beta^{*}$ leveling with telescopic ATS squeeze (MD 2410), Sep 2017. URL: https://cds.cern.ch/record/ 2285184.

[6] M. Hostettler, A. Calia, K. Fuchsberger, M. Gabriel, G.-H. Hemelsoet, M. Hruska, D. Jacquet, and J. Wenninger. $\beta^{*}$ levelling using the LHC Lumi Server (MD 2427), Jan 2018. URL: https://cds.cern.ch/record/2300212.

[7] G. Apollinari, I. Béjar Alonso, O. Brüning, M. Lamont, and L. Rossi, editors. $H L-L H C$ Preliminary Design Report: Deliverable: D1.5. CERN, Geneva, Nov 2014. CERN-ACC-20140300. URL: http://cds.cern.ch/record/1972604.

[8] G. Rumolo, A. Axford, G. Iadarola, and A. Romano. Bunch length and particle distribution for (HL-)LHC. 48th HL-LHC WP2 Meeting, CERN, Geneva, Apr 2015. URL: https://indico.cern.ch/event/376196/contribution/2/attachments/ 749822/1028685/HL-LHCTaskLeader-GR-240415.pdf.

[9] R. Tomás, O. Dominguez, and S. White. HL-LHC alternatives. In B. Goddard and F. Zimmermann, editors, Proc. of RLIUP: Review of LHC and Injector Upgrade Plans, Archamps, France, October 29-31, 2013, CERN Yellow Reports: Conference Proceedings, pages 119-126, Geneva, Switzerland, 2014. CERN. CERN-2014-006. URL: https://cds.cern.ch/record/ 1977364, doi:10.5170/CERN-2014-006.119.

[10] R. Tomás, G. Arduini, D. Banfi, J. Barranco, H. Bartosik, O. Brüning, R. Calaga, O. Dominguez, H. Damerau, S. Fartoukh, S. Hancock, G. Iadarola, R. De Maria, E. Métral, T. Pieloni, G. Rumolo, B. Salvant, E. Shaposhnikova, and S. White. HL-LHC alternative scenarios. In M. Draper, editor, Proc. of Chamonix 2014 Workshop on LHC Performance, Chamonix, France, September 22-25, 2014, CERN Yellow Reports: Conference Proceedings, pages 217-224, Geneva, Switzerland, 2015. CERN. CERN-2015-002; CERN-ATS-2015-002. URL: https://cds. cern.ch/record/2031210.

[11] R. Calaga and R. Tomás. A 200 MHz SC-RF system for the HL-LHC. In Proc. of International Particle Accelerator Conference (IPAC'16), Busan, Korea, May 8-13, 2016, pages 1513-1515, Geneva, Switzerland, Jun 2016. JACoW. URL: http://jacow.org/ipac2016/ papers/tupmw034.pdf. 
[12] P. Azzi. CMS performance: Dependence on scenario for luminous region. ECFA High Luminosity LHC Experiments Workshop 2016, Aix-Les-Bains, France, Oct 2016. URL: https://indico.cern.ch/event/524795/contributions/2235253/ attachments/1347082/2031647/EDQCMS_ECFA_Azzi.pdf.

[13] B. Petersen. ATLAS performance for different luminous region scenarios. ECFA High Luminosity LHC Experiments Workshop 2016, Aix-Les-Bains, France, Oct 2016. URL: https://indico.cern.ch/event/524795/contributions/2235251/attachments/ 1347098/2031687/LumiStudies.pdf.

[14] S. Fartoukh. Pile up management at the high-luminosity LHC and introduction to the crabkissing concept. Phys. Rev. ST Accel. Beams, 17:111001, Nov 2014. URL: https://link. aps.org/doi/10.1103/PhysRevSTAB.17.111001, doi:10.1103/PhysRevSTAB.17.111001.

[15] ATLAS Collaboration. Technical design report for the ATLAS inner tracker strip detector. Technical Report CERN-LHCC-2017-005, ATLAS-TDR-025, CERN, Geneva, Apr 2017. URL: http://cds.cern.ch/record/2257755.

[16] B. Petersen. ATLAS pile-up studies. 9th Experimental Data Quality Meeting, CERN, Geneva, Apr 2017. URL: https://indico.cern.ch/event/626264/contributions/ 2529218/attachments/1442395/2221239/AtlasStudies.pdf.

[17] B. Petersen. Private communication.

[18] R Tomás. Updated optics layout and machine performance. 6th HL-LHC Collaboration Meeting, Paris, France, Nov 2016. URL: https://indico.cern.ch/event/549979/ contributions/2257122/attachments/1370681/2079634/SLIDESBeamer . pdf.

[19] E. Shaposhnikova and J.E. Muller. Bunch length and particle distribution for (HL-)LHC. 82nd HL-LHC WP2 Meeting, CERN, Geneva, Jan 2017. URL: https://indico.cern.ch/event/572439/contributions/2423329/attachments/ 1394442/2125205/HL-LHC_WP2_Jan17.pdf.

[20] J.F. Esteban Müller. Longitudinal intensity effects in the CERN Large Hadron Collider. PhD thesis, EPFL, Lausanne, 2016. URL: https://infoscience.epfl.ch/record/219008/ files/EPFL_TH7077.pdf, doi:10.5075/epfl-thesis-7077.

[21] V. Shiltsev and E. McCrory. Characterizing luminosity evolution in the Tevatron. FERMILAB-CONF-05-191-AD, 2005. URL: http://lss.fnal.gov/archive/2005/conf/ fermilab-conf-05-191-ad.pdf. 
[22] M. Giovannozzi. Simple models describing the time-evolution of luminosity in hadron colliders. CERN-ACC-2014-0086, Jun 2014. URL: https://cds.cern.ch/record/1742039.

[23] ATLAS and CMS Collaborations. Expected pileup values at the HL-LHC. Technical Report ATL-UPGRADE-PUB-2013-014, CERN, Geneva, Sep 2013. URL: https://cds.cern.ch/ record/1604492.

[24] M. Martini. Intrabeam scattering. CERN Accelerator School: Intensity Limitations in Particle Beams, CERN, Nov 2015. URL: https://cas.web.cern.ch/cas/ Intensity-Limitations-2015/Lectures/Saturday7/Martini.pdf.

[25] MAD - Methodical Accelerator Design, May 2018. URL: http://mad.web.cern.ch/mad/.

[26] G. Arduini, D. Banfi, J. Barranco, R. Bruce, O. Brüning, R. De Maria, O. Dominguez, P. Fessia, M. Fitterer, S. Gilardoni, M. Giovannozzi, B. Gorini, G. Iadarola, K. Kain, M. Kuhn, E. Métral, N. Mounet, T. Pieloni, S. Redaelli, L. Rossi, G. Rumolo, R. Tomás, J. Wenninger, and A. Valishev. PICs: what do we gain in beam performance. LIU-HL-LHC: PICs: what do we gain in beam performances? In S. Myers and F. Zimmermann, editors, Review of LHC and injector upgrade plans - Summary. CERN, Oct 2013. CERN-ACC-NOTE-2014-0071. URL: https://cds.cern.ch/record/1953694.

[27] L.E. Medina Medrano, G. Arduini, and R. Tomás. Studies on luminous region, pile-up and performance for HL-LHC scenarios. In Proc. of International Particle Accelerator Conference (IPAC'17), Copenhagen, Denmark, 14-19 May, 2017, pages 1908-1911, Geneva, Switzerland, May 2017. JACoW. URL: http://jacow.org/ipac2017/papers/tupik089.pdf.

[28] R. Tomás. HL-LHC operational scenarii and machine performance. 7th HL-LHC Collaboration Meeting, Paris, France, Nov 2017. URL: https://indico.cern.ch/event/647714/ contributions/2632846/attachments/1556811/2450371/SLIDESlogo.pdf.

[29] E. Métral, S. Antipov, F. Antoniou, R.B. Appleby, G. Arduini, J. Barranco García, P. Baudrenghien, N. Biancacci, C. Bracco, R. Bruce, X. Buffat, R. Calaga, L.R. Carver, E. Chapochnikova, M.P. Crouch, R. De Maria, S. Fartoukh, D. Gamba, M. Giovannozzi, P. Gonçalves Jorge, W. Hofle, G. Iadarola, N. Karastathis, A. Lasheen, T. Mastoridis, L.E. Medina Medrano, A. Mereghetti, D. Mirarchi, B. Muratori, P.S. Papadopoulou, Y. Papaphilippou, D. Pellegrini, T. Pieloni, S. Redaelli, G. Rumolo, B. Salvant, M. Solfaroli Camillocci, C. Tambasco, R. Tomas García, and D. Valuch. Update of the HL-LHC operational scenarios for proton operation. CERN-ACC-2014-0086, Jan 2018. URL: http: 
//cds.cern.ch/record/2301292.

[30] G. Apollinari, I. Béjar Alonso, O. Brüning, M. Lamont, and L. Rossi, editors. High-Luminosity Large Hadron Collider (HL-LHC) Technical Design Report. CERN, Geneva, Dec 2015. CERN2015-005, unpublished.

[31] L. Rossi. HL-LHC: baseline and operational scenario. LHC Performance Workshop Chamonix, Jan 201/. URL: https://indico.cern.ch/event/676124/contributions/ 2768608/attachments/1591920/2520437/1_-_HL-LHC_Baseline_and_recap_options_-_ Rossi.pdf.

[32] L.E. Medina Medrano and R. Tomás. Performance and operational aspects of HL-LHC scenarios. In Proc. of International Particle Accelerator Conference (IPAC'16), Busan, Korea, May 8-13, 2016, pages 1516-1519, Geneva, Switzerland, Jun 2016. JACoW. URL: http://jacow.org/ipac2016/papers/tupmw035.pdf.

[33] S. Papadopoulou, F. Antoniou, T. Argyropoulos, M. Fitterer, M. Hostettler, and Y. Papaphilippou. Modelling and measurements of bunch profiles at the LHC. In Proc. of International Particle Accelerator Conference (IPAC'17), Copenhagen, Denmark, 14-19 May, 2017, pages 2167-2170, Geneva, Switzerland, May 2017. JACoW.

[34] S. Umarov, C. Tsallis, and S. Steinberg. On a q-central limit theorem consistent with nonextensive statistical mechanics. Milan Journal of Mathematics, 76(1):307-328, 2008. URL: http://dx.doi.org/10.1007/s00032-008-0087-y, doi:10.1007/s00032-008-0087-y.

[35] F.S. Carlier, J.M. Coello De Portugal Martinez Vazquez, S. Fartoukh, E. Fol, D. Gamba, A. Garcia-Tabares Valdivieso, M. Giovannozzi, M. Hofer, A.S. Langner, E.H. Maclean, L. Malina, L.E. Medina Medrano, T.H.B. Persson, P.K. Skowronski, R. Tomas Garcia, F. Van Der Veken, and A. Wegscheider. Optics measurements and correction challenges for the HLLHC, Oct 2017. URL: https://cds. cern.ch/record/2290899.

[36] S. Fartoukh, A. Valishev, Y. Papaphilippou, and D. Shatilov. Compensation of the long-range beam-beam interactions as a path towards new configurations for the high luminosity LHC. Phys. Rev. ST Accel. Beams, 18:121001, Dec 2015. URL: https://link.aps.org/doi/10. 1103/PhysRevSTAB.18.121001, doi:10.1103/PhysRevSTAB.18.121001.

[37] J. Barranco García and T. Pieloni. Global compensation of long-range beam-beam effects with octupole magnets: dynamic aperture simulations for the HL-LHC case and possible usage in LHC and FCC. CERN-ACC-NOTE-2017-0036, May 2017. URL: https://cds.cern.ch/ 
record/2263347.

[38] L.E. Medina Medrano, J. Barranco García, X. Buffat, Y. Papaphilippou, T. Pieloni, and R. Tomás. Correction of beta-beating due to beam-beam for the LHC and its impact on dynamic aperture. In Proc. of International Particle Accelerator Conference (IPAC'17), Copenhagen, Denmark, 14-19 May, 2017, pages 2512-2515, Geneva, Switzerland, May 2017. JACoW. URL: http://jacow.org/ipac2017/papers/weoab2.pdf.

[39] E.N. Shaposhnikova and J.F. Esteban Müller. Possible beam parameters in double RF operation of the CERN LHC. In Proc. of International Particle Accelerator Conference (IPAC'16), Busan, Korea, May 8-13, 2016, pages 2430-2433, Geneva, Switzerland, June 2016. JACoW. URL: http://jacow.org/ipac2016/papers/wepmw008.pdf.

[40] G. Iadarola and G. Rumolo. $200 \mathrm{MHz}$ option for HL-LHC: e-cloud considerations (heat load aspects). 68th HL-LHC WP2 Meeting, CERN, Geneva, May 2016. URL: https://indico.cern.ch/event/523881/contributions/2154409/ attachments/1267275/1876504/006_ecloud_200Mhz.pdf.

[41] K. Li and E. Métral. $200 \mathrm{MHz}$ option for HL-LHC and intensity limitations. 68th HL-LHC WP2 Meeting, CERN, Geneva, May 2016. URL: https://indico.cern.ch/event/523881/ contributions/2154410/attachments/1267263/1876558/001b_200_MHZ_option.pdf.

[42] F.-J. Decker. Beam distributions beyond RMS. SLAC-PUB-6684, Sep 1994. URL: http: //www.slac.stanford.edu/pubs/slacpubs/6500/slac-pub-6684.pdf.

[43] K. Ohmi, R. Tomás, Y. Funakoshi, R. Calaga, T. Ieiri, Y. Morita, K. Nakanishi, K. Oide, Y. Ohnishi, Y. Sun, M. Tobiyama, and F. Zimmermann. Response of colliding beam-beam system to harmonic excitation due to crab-cavity rf phase modulation. Phys. Rev. ST Accel. Beams, 14:111003, Nov 2011. URL: https://link.aps.org/doi/10.1103/PhysRevSTAB. 14 . 111003, doi:10.1103/PhysRevSTAB.14.111003.

[44] P. Baudrenghien and T. Mastoridis. Transverse emittance growth due to rf noise in the high-luminosity LHC crab cavities. Phys. Rev. ST Accel. Beams, 18:101001, Oct 2015. URL: https://link.aps.org/doi/10.1103/PhysRevSTAB .18.101001, doi:10.1103/ PhysRevSTAB.18.101001.

[45] P. Baudrenghien, R. Calaga, T. Mastoridis, and E. Yamakawa. Crab cavities, Rf noise and operational aspects (counter-phasing, full detuning). An update. 96th HL-LHC WP2 Meeting, CERN, Geneva, Jun 2017. URL: https://indico.cern.ch/event/645814/contributions/ 
2622537/attachments/1475139/2291024/Meeting_13_06_2017.pdf.

[46] T. Mastoridis, P. Baudrenghien, S. Hansen, B. Miller, and J. Wachter. Crab cavity RF noise mitigation. 101st HL-LHC WP2 Meeting, CERN, Geneva, Aug 2017. URL: https://indico.cern.ch/event/659970/contributions/2692112/ attachments/1510812/2355979/ABPtalk_Aug2017.pdf. 